\title{
Review
}

Karin Nagy*, Ina Skagervik, Hayrettin Tumani, Axel Petzold, Manfred Wick, Hans-Jürgen Kühn, Manfred Uhr, Axel Regeniter, Johannes Brettschneider, Markus Otto, Jörg Kraus, Florian Deisenhammer, Ronald Lautner, Kaj Blennow, Leslie Shaw, Henrik Zetterberg and Niklas Mattsson

\section{Cerebrospinal fluid analyses for the diagnosis of subarachnoid haemorrhage and experience from a Swedish study. What method is preferable when diagnosing a subarachnoid haemorrhage?}

\begin{abstract}
Subarachnoid haemorrhage (SAH) has a high mortality and morbidity rate. Early SAH diagnosis allows the early treatment of a ruptured cerebral aneurysm, which improves the prognosis. Diagnostic cerebrospinal fluid (CSF) analyses may be performed after a negative computed tomography scan, but the precise analytical methods to be used have been debated. Here, we summarize the scientific evidence for different CSF methods for SAH diagnosis and describe their implementation in different countries. The principle literature search was conducted using PubMed and Scopus with the search items "cerebrospinal fluid", "subarachnoid haemorrhage", and "diagnosis". CSF analyses for SAH include visual examination, red blood cell counts, spectrophotometry for oxyhaemoglobin or bilirubin determination, CSF cytology, and ferritin measurement. The methods vary in availability and performance. There is a consensus that spectrophotometry has the highest diagnostic performance, but both oxyhaemoglobin and bilirubin determinations are susceptible to important confounding factors. Visual inspection of CSF for xanthochromia is still frequently used for diagnosis of $\mathrm{SAH}$, but it is advised against because spectrophotometry has a superior diagnostic accuracy. A positive finding of CSF bilirubin is a strong indicator of an intracranial bleeding, whereas a positive finding of CSF oxyhaemoglobin may indicate an intracranial bleeding or a traumatic tap. Where spectrophotometry is not available, the combination of CSF cytology for erythrophages or siderophages and ferritin is a promising alternative.
\end{abstract}

Keywords: bilirubin; cerebrospinal fluid; diagnosis; haemoglobin; subarachnoid haemorrhage.
*Corresponding author: Karin Nagy, MD, Clinical Neurochemistry Laboratory, Sahlgrenska University Hospital/Mölndal, S-431 80 Mölndal, Sweden, E-mail: karin.nagy@gmail.com

Ina Skagervik: Department of Neurosurgery, Sahlgrenska University Hospital, Gothenburg, Sweden

Hayrettin Tumani, Johannes Brettschneider and Markus Otto: Department of Neurology, University of Ulm, Ulm, Germany Axel Petzold: University College London Institute of Neurology, Department of Neuroimmunology, National Hospital for Neurology and Neurosurgery, London, UK; Department of Neurology and VU University Medical Center, Department of Neurology, Amsterdam, The Netherlands

Manfred Wick: Department of Clinical Chemistry, Ludwig Maximilian University, Munich, Germany

H.-J. Kühn: Department of Clinical Chemistry, University of Leipzig, Leipzig, Germany

Manfred Uhr: Max Planck Institute for Psychiatry, Munich, Germany Axel Regeniter: Department of Clinical Chemistry, University Hospital Basel, Basel, Switzerland

Jörg Kraus: Department of Neurology, Paracelsus Medical University, Salzburger Landesklinik, Salzburg, Austria Florian Deisenhammer: Department of Neurology, University of Innsbruck, Innsbruck, Austria Ronald Lautner, Kaj Blennow, Henrik Zetterberg and Niklas Mattsson: Clinical Neurochemistry Laboratory, Institute of Neuroscience and Physiology, Department of Psychiatry and Neurochemistry, Sahlgrenska Academy, University of Gothenburg, Gothenburg/Mölndal, Sweden

Leslie Shaw: Department of Pathology and Laboratory Medicine, University of Pennsylvania Medical Center, Philadelphia, PA, USA

\section{Introduction}

Subarachnoid haemorrhage (SAH) accounts for approximately $5 \%$ of all strokes and has a yearly incidence of approximately 1 in 10,000 persons [1]. Importantly, SAH both has a high fatality (30\%-70\%) [2] and may occur at 
an early age compared with other causes of stroke [3]. In the majority of cases, SAH is caused by a spontaneous rupture of an aneurysm in the arterial circulation of the brain. Typical initial symptoms are severe headache with a sudden onset ("thunderclap headache"), neck stiffness, vomiting, confusion, or loss of consciousness. Tools for SAH diagnosis include imaging and cerebrospinal fluid (CSF) analyses. There is no definite international consensus on a preferred diagnostic algorithm, and procedures differ between countries and regions, which may have an impact on patients in terms of diagnosis, treatment, and prognosis. In this review, we aim to discuss available CSF methods for SAH diagnosis, clarify their strengths and weaknesses, and outline their use on a global scale, focusing on recent guidelines and experiences from laboratory medicine in the UK, Germany, Sweden, the Netherlands, Austria, and the USA.

\section{Methods}

The literature search for this review was based on the intention to include all up-to-date studies to accurately describe the current scientific basis for CSF-based diagnostics of SAH and to illustrate the variability in methods between countries. We performed searches on PubMed with the principal search items "cerebrospinal fluid", "subarachnoid haemorrhage", and "diagnosis". We did additional searches with the added keywords "spectrophotometry", "oxyhaemoglobin", "methaemoglobin”, "bilirubin”, "ferritin", and "siderophages". The database Scopus was used to identify more recent publications citing selected references. From identified references, we included relevant clinical studies, review articles, editorials, and comments. Selection criteria included a judgment on the importance of the studies, where prospective studies, large case series, guidelines, and consensus documents were prioritized. The general search was restricted to articles in English published after 1990, but since we aimed to review methods across different countries, we also included specifically selected articles written in other languages. We also examined older important publications that we were previously aware of. Finally, we also here present novel data from a Swedish control survey of SAH diagnostics.

\section{The importance of early diagnosis}

An incorrect initial diagnosis of SAH patients is common and is associated with worse prognosis [4]. SAH patients may initially present with less serious clinical symptoms (called sentinel headache) caused by a small bleeding (warning leak), followed by a manifest SAH, often within 2 weeks [5]. Sentinel headache has been reported in up to $40 \%$ of SAH patients [6]. It is of uttermost importance to identify these patients because interventional therapy at this stage may hinder a life-threatening bleeding and significantly improve prognosis [7-9].

\section{Diagnostic methods}

Clinical examination is insufficient for SAH diagnosis. The most commonly used first line of investigation is noncontrast computed tomography (CT) of the brain. CT has an excellent sensitivity for SAH in the acute stage, especially using modern scanners, with sensitivities up to $98 \%-100 \%$ within the first hours from symptom onset [10, 11]. However, subtle abnormalities may not be detected by inexperienced or nonspecialized physicians, and expert neuroradiologists are not available at all hospitals [4]. However, because subarachnoid blood is rapidly cleared from the brain, the sensitivity drops quickly. Six hours after onset, the sensitivity of a CT scan for finding an SAH is approximately 86\%-93\% $[11,12]$, and it decreases to approximately $50 \%$ after 1 week (Table 1) [13]. Importantly, these figures are likely to be even lower when scans are evaluated by nonspecialized radiologists, as is often the case in smaller hospitals or outside business hours. A conventional cerebral angiography is usually performed on patients with a positive CT scan to locate a possible aneurysm for interventional therapy. For patients with a negative CT scan where the clinical suspicion remains strong, more investigations are needed [20]. Fluid attenuated inversion recovery (FLAIR) magnetic resonance imaging (MRI) may have higher sensitivity than CT [21], but in clinical practice, the choice after a negative CT scan most often stands between a conventional cerebral angiography and a lumbar puncture (LP) for CSF analyses. A cerebral angiography is resource demanding, carries a slight risk for stroke as a complication, and may result in false-positive results and unwarranted interventions because incidental aneurysms are found in approximately $5 \%$ of the population [22]. These factors usually make CSF analyses preferred as a second-line of investigation after a negative CT scan.

\section{CSF analyses}

There are several widely used CSF analyses for SAH, with different diagnostic performance, availability, and 
Table 1 Sensitivity and specificity for CT and biomarkers when diagnosing SAH.

\begin{tabular}{|c|c|c|c|c|}
\hline Biomarker method & Time since ictus & Sensitivity, \% & Specificity, \% & Reference \\
\hline CT & $<6 \mathrm{~h}$ & $98-100$ & 100 & {$[10,11]$} \\
\hline $\mathrm{CT}$ & $>6 \mathrm{~h}$ & $86-93$ & 100 & {$[11,12]$} \\
\hline CT & $>1$ week & 50 & $\begin{array}{l}\text { High, but accurate } \\
\text { numbers are missing }\end{array}$ & [13] \\
\hline $\begin{array}{l}\text { Visual inspection for } \\
\text { xantochromia }\end{array}$ & $>12 \mathrm{~h}$ & $27-47$ & 98 & {$[14,15]$} \\
\hline Red blood cell count & $\begin{array}{l}\text { Early in the progress or } \\
\text { due to a traumatic tap }\end{array}$ & $\begin{array}{l}\text { High, but accurate numbers } \\
\text { are missing }\end{array}$ & $\begin{array}{l}\text { Low, due to high } \\
\text { false-positive rate }\end{array}$ & \\
\hline $\begin{array}{l}\text { Oxyhaemoglobin, } \\
\text { spectrophotometry }\end{array}$ & Not specified & 84 & 42 & [16] \\
\hline Bilirubin, spectrophotometry & Not specified & $83-100$ & $75-88$ & {$[16,17]$} \\
\hline $\begin{array}{l}\text { Methaemoglobin, } \\
\text { spectrophotometry }\end{array}$ & Not specified & 66 & 79 & [16] \\
\hline Ferritin $>15 \mathrm{ng} / \mathrm{mL}$ & Not specified & 95 & 95 & [18] \\
\hline Siderophages & $>24 \mathrm{~h}$ & $\begin{array}{l}\text { High, but accurate numbers } \\
\text { are missing }\end{array}$ & $\begin{array}{l}\text { High, but accurate } \\
\text { numbers are missing }\end{array}$ & [19] \\
\hline
\end{tabular}

technical requirements. A consequence in clinical practice of differences between methods and how they are implemented is that patients may receive different diagnoses and treatments at different hospitals.

\section{Visual inspection}

The most basic CSF method for SAH is the visual evaluation of discoloration. For this, the sample is usually centrifuged and the supernatant compared with a tube of water, held against a well-illuminated white background. Normal CSF is uncolored, and transparent. After an SAH, a yellowish or reddish tint is seen due to bilirubin (called xanthochromia) or oxyhaemoglobin. According to a survey from 2002, most US laboratories use visual assessment of xanthochromia as their main CSF analysis of SAH [23]. However, the sensitivity of the human color vision is not sufficient to detect a very small amount of bilirubin, which may appear as transparent as water [14, 24]. The human eye also has difficulty in assessing xanthochromia of a predominantly red fluid, which makes it problematic to visually distinguish a red sample after a traumatic tap from a bilirubin-rich sample after an SAH. A traumatic tap may occur when the LP needle punctuates a blood vessel. This has been estimated to happen in 10\%-20\% of LPs but may be even more frequent depending on the definitions of the cutoff towards a normal CSF erythrocyte count [25-29]. A traumatic tap introduces erythrocytes in the CSF, which may undergo in vitro lysis and release their oxyhaemoglobin content. On visual inspection, approximately $80 \%$ of CSF samples with significant bilirubin levels seem red rather than yellow due to the presence of oxyhaemoglobin [30]. In sum, visual inspection has poor diagnostic sensitivity for SAH (Table 1) $[14,15]$ and a higher proportion of laboratory technicians identify bilirubin when using a spectrophotometer compared with visual inspection [24, 31]. The subjectivity of visual evaluation also makes it difficult to perform comparisons over time. There are also other possible causes of CSF discoloration, as explained in the Confounding Factors section.

\section{Red blood cell count}

In principle, normal CSF should not contain erythrocytes, but the red blood cell count expected in CSF after an SAH, differentiating it from a traumatic tap, is not well defined. Cell counts are sometimes performed on serial tubes, to detect declining red blood cell counts, indicating a traumatic tap (the so-called three-tube method). However, because a traumatic tap may result in discontinuing and resuming bleedings and may occur also in the presence of an SAH, the serial tube method is unreliable [32, 33].

\section{Spectrophotometry}

Spectrophotometry is used to detect the absorption spectrum of CSF pigments, and the presence of different biomarkers may be estimated from the absorption curve. It is a rapid ( $<1 \mathrm{~min})$ technique that requires a small sample amount (50-500 $\mu \mathrm{L})$ [28]. Because sensitivity and 
specificity vary for different markers that may be elevated depending on the duration since symptom onset, the laboratory technician must know the time point of symptom debut, sampling, and centrifugation to perform a sound interpretation (Table 1).

\section{Oxyhaemoglobin}

After an SAH, the erythrocytes in the CSF will gradually lyse and release their intracellular haemoglobin. This process starts shortly $(2-4 \mathrm{~h})$ after the bleeding, and oxyhaemoglobin can be detected in CSF from approximately 2-12 h after ictus [34]. Oxyhaemoglobin, the oxygenated form of haemoglobin, has an absorbance maximum around $415 \mathrm{~nm}$, in the violet region of the optical spectrum (Figure 1A) [28]. Minor secondary absorbance peaks, sometimes referred to as Soret bands, may be detected at 540 and $580 \mathrm{~nm}$, in the green region of the optical spectrum.

\section{Bilirubin}

Oxyhaemoglobin is metabolized to bilirubin through enzymatic reactions by macrophages in vivo. This does not occur in vitro in tapped CSF (except in samples with very high white blood cell count), which strongly reduces the risk for false-positive results caused by a traumatic tapping. Bilirubin has a broad maximum absorption peak in the blue region of the optical spectrum at $450-460 \mathrm{~nm}$ [28]. On spectrophotometry of an acute bleeding, where oxyhaemoglobin is still present in high concentrations, bilirubin may be visible as a gentle slope to the right of the sharp oxyhaemoglobin peak (Figure 1B). As an alternative to using a spectrophotometer, CSF bilirubin may also be measured by the modification of the direct diazo methods used for bilirubin measurements in serum or plasma. This has been suggested as a screening instrument to identify samples that need to be further analyzed by spectrophotometry [35, 36], but difficulties regarding technical analyses and method standardization may hinder a broad implementation of this tentative approach [37].

\section{Methaemoglobin}

Methaemoglobin is haemoglobin with ferric $\left(\mathrm{Fe}^{3+}\right)$ instead of ferrous iron $\left(\mathrm{Fe}^{2+}\right)$ in the haem group, with a broad absorbance maximum peak at 403-410 nm. Normally, methaemoglobin is present in very small amounts, but it has been reported in CSF of patients with subdural
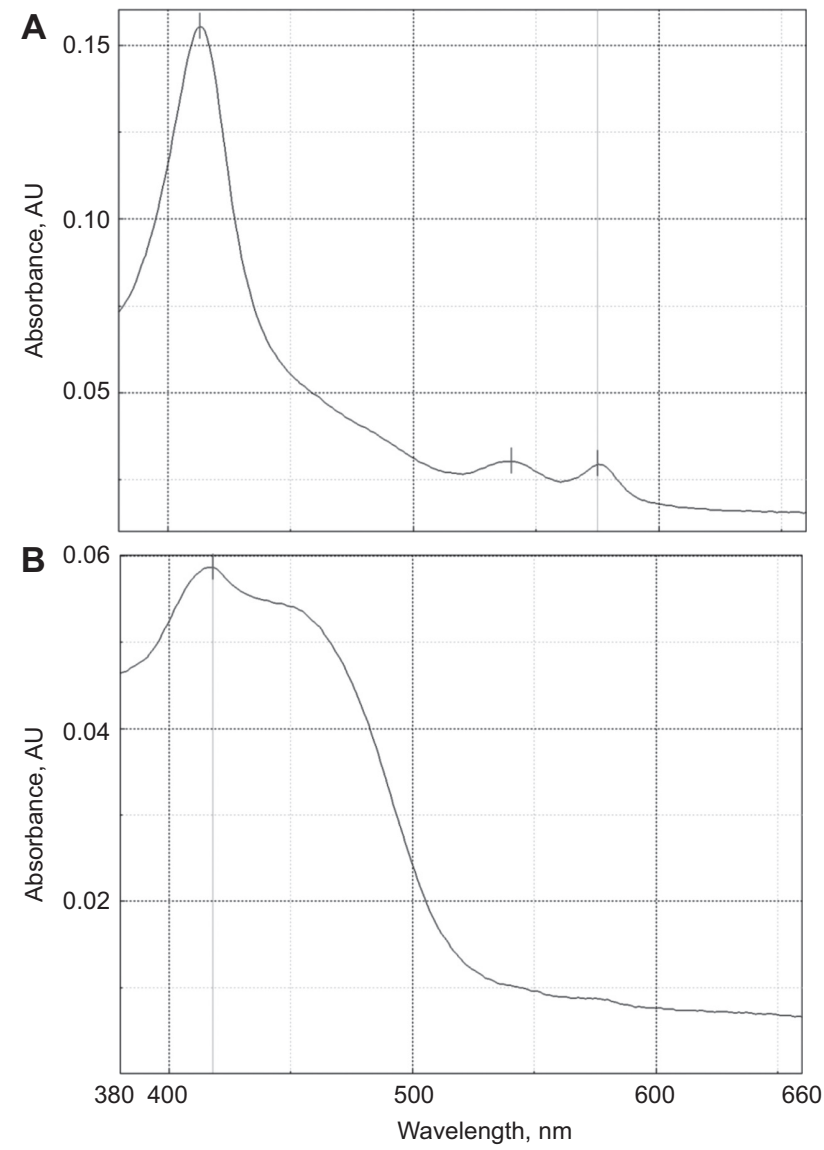

Figure 1 Spectrophotometry of oxyhaemoglobin (A) and bilirubin (B) slope.

haematoma or an enclosed bleeding, giving the CSF a brownish color [38]. A spontaneous oxidation of the haem group occurs around 10 days after a bleed, irrespective of cause [39], which may be useful to distinguish between a traumatic tap and a cerebral haemorrhage [40].

\section{Ferritin}

Ferritin is an iron-storage protein complex that removes toxic iron released from the metabolized haemoglobin. The production of this large protein takes some time, and therefore, CSF ferritin has a lower sensitivity in the first hours after SAH and is not advocated for diagnostic use at an early stage [41, 42]. A first increase is possibly due to the activation of macrophages. It takes approximately 3 days for the CSF ferritin levels to consistently increase and 7-10 days to reach over $1000 \mathrm{ng} / \mathrm{mL}$ [43]. For comparison, control CSF has been reported to have an average ferritin level of approximately $4 \mathrm{ng} / \mathrm{mL}$, whereas CSF contaminated by a traumatic LP has been reported to have an average ferritin level of approximately $9 \mathrm{ng} / \mathrm{mL}$ [43]. 
One conservative reference limit that has been proposed is $12 \mathrm{ng} / \mathrm{mL}$ [44], and in a prospective study, a cutoff of $15 \mathrm{ng} / \mathrm{mL}$ achieved $95 \%$ sensitivity and specificity for SAH (Table 1) [18]. Importantly, CSF ferritin levels remain high long after CSF bilirubin decrease or become undetectable $[42,43]$.

\section{Siderophages}

SAH induces an inflammatory response that includes macrophage activation. Macrophages that have consumed erythrocytes develop haemosiderin storage and are called siderophages. Cytological finding of CSF siderophages have a low sensitivity within the first 3 days after an intracranial bleeding, making them unsuitable for early diagnosis, but later in the process, they have high sensitivity and specificity, and they may even be detected for several months after an insult (Table 1) [32, 19]. In combination with erythrophages (macrophages that do not have haemosiderin inside), they can give important clues about the age and dynamics of the bleeding [45].

\section{Other methods}

Other CSF biomarkers tested for SAH diagnosis require further evaluation (for example, urine test stripes for rapid detection of red blood cells in CSF [46]) or have shown inconclusive results (for example, CSF D-dimer [12, 47, 48]).

\section{Regional practices}

The implementation of the methods used to diagnose SAH after a negative CT scan outlined above differs between countries and regions, even among specialized centers. Here, we experiences from different countries, focusing on official UK guidelines, a recently completed survey among Swedish laboratories, and the authors own experiences from practice in Germany, Austria, Switzerland, and USA.

\section{United Kingdom}

The UK National External Quality Assessment Service (NEQAS) guidelines stress the use of spectrophotometry and have reduced the use of visual assessment for SAH diagnosis in the UK $[49,50]$. These guidelines emphasize the use of bilirubin because this is solely produced in vivo, as opposed to oxyhaemoglobin and methaemoglobin [50], reducing the risks for false-positive results. Accordingly, the UK guidelines state that the sampling should be no $<12 \mathrm{~h}$ after the possible bleeding. The UK guidelines advocate scanning between 350 and $600 \mathrm{~nm}$ to allow interpretation of the curve for bilirubin, oxyhaemoglobin, and methaemoglobin (which is considered a probable artefact of oxyhaemoglobin conversion and given the same significance as oxyhaemoglobin). The net bilirubin absorbance (NBA) is calculated according to Chalmers' modified method [51], but the laboratory technician should also measure the net oxyhaemoglobin absorbance (NOA) at the maximum oxyhaemoglobin absorbance. The absence of bilirubin and oxyhaemoglobin $(\mathrm{NBA} \leq 0.007 \mathrm{AU}$ and NOA $\leq 0.02 \mathrm{AU}$ ) is reported as "no evidence to support SAH". Small amounts of oxyhaemoglobin (NBA $\leq 0.007 \mathrm{AU}$ and $0.1 \mathrm{AU}>\mathrm{NOA}>0.002 \mathrm{AU}$ ) is also reported as "no evidence to support SAH", but an oxyhaemoglobin peak large enough to allow impairment of bilirubin detection ( $\mathrm{NBA} \leq 0.007 \mathrm{AU}$ and $\mathrm{NOA} \geq 0.1 \mathrm{AU}$ ) is reported as "SAH not excluded". If bilirubin is detected alone (NBA $>0.007 \mathrm{AU}$ and NOA $\leq 0.02 \mathrm{AU}$ or NOA $>0.02$ but with no visible oxyhaemoglobin peak), the laboratory technician considers the serum bilirubin and CSF protein levels because these may give false-positive bilirubin results. If serum bilirubin is low $(\leq 20 \mu \mathrm{mol} / \mathrm{L})$, the result is reported as "consistent with SAH", and if serum bilirubin is high ( $>20 \mu \mathrm{mol} / \mathrm{L})$, the NBA is adjusted accordingly and reported as "consistent with SAH" (if still increased) or "not supportive of SAH" (if the increase depended on serum bilirubin). Increased bilirubin (NBA $>0.007 \mathrm{AU}$ ) in the presence of elevated CSF protein levels $(>1.0 \mathrm{~g} / \mathrm{L})$ is reported as a possible $\mathrm{SAH}$, but other sources of increased CSF bilirubin must then also be considered. Finally, a concurrent finding of bilirubin and oxyhaemoglobin (NBA>0.007 AU and NOA $>0.02 \mathrm{AU}$ with visible oxyhaemoglobin peak) is reported as "consistent with SAH". This scheme has become influential, but its use is not trivial, and it requires trained staff and a substantial through-flow of samples to maintain observer skills.

\section{Sweden}

During 2010, a survey of CSF analyses in SAH investigations in Sweden was performed. A questionnaire was sent out to all Swedish laboratories participating in quality control programs organized by the External Quality Assurance in Laboratory Medicine in Sweden (EQUALIS) $(n=24)$. Twenty-two laboratories reported that they used 
spectrophotometry as their main method. Ten of these measured absorbance at $415 \mathrm{~nm}$ (with or without a full curve); five laboratories stated that they used spectrophotometry but did not further specify how; five laboratories followed the UK NEQAS guidelines; two laboratories measured absorbance at $415 \mathrm{~nm}$ plus direct measurement of CSF bilirubin; and two laboratories evaluated the samples visually and did cell counts. To investigate if these differences may result in different patient management, two survey samples were sent out (prepared at the Clinical Neurochemistry Laboratory in Mölndal by deidentified CSF samples from the routine workflow). Each sample had a (fictional) medical history. The first sample was presented as "male, 34 years old, acute headache, debuted $8 \mathrm{~h}$ before sampling. Bleeding?” Spectrophotometry at Mölndal showed a distinct oxyhaemoglobin peak (absorbance, $415 \mathrm{~nm}, 0.16 \mathrm{AU}$; Figure 2A). Of 17 responding laboratories, 11 reported that SAH could not be excluded (9 specifically answered that the result also was consistent with a traumatic tap), three reported that the result indicated SAH, and two reported that SAH was unlikely. The second sample was presented as "woman, 66 years old, has been feeling uncomfortable since 2-3 days. In the beginning, she also had a headache. Bleeding?"
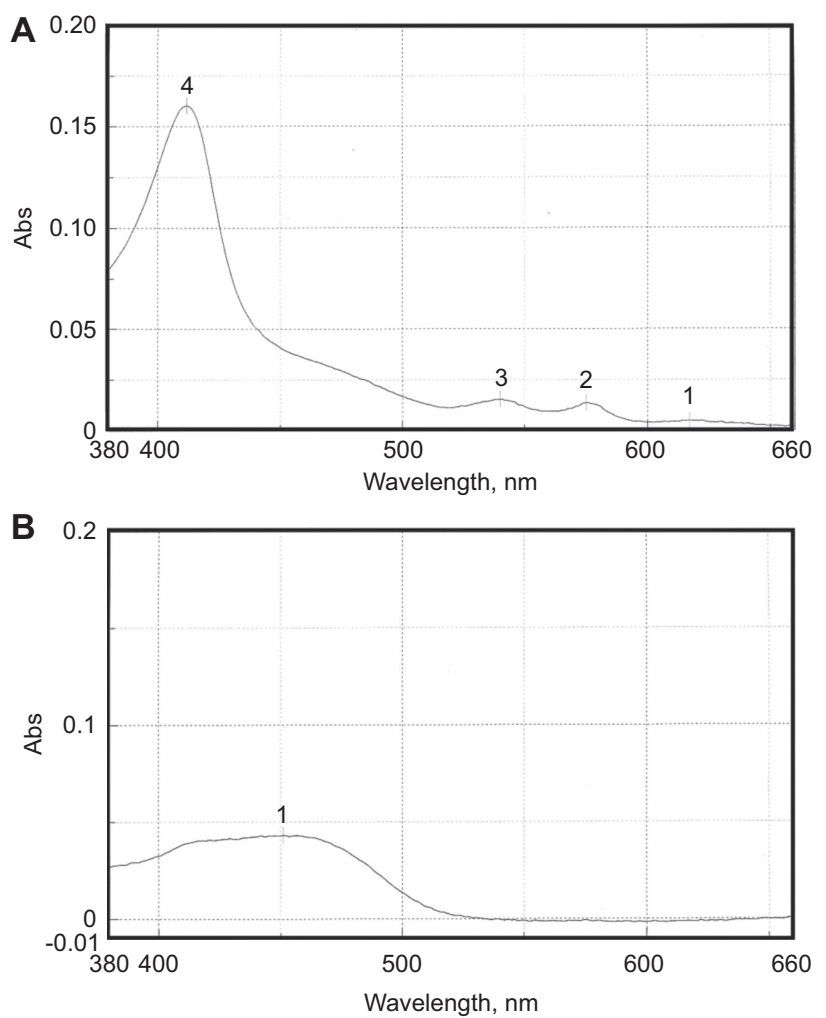

Figure 2 Spectrophotometry of samples from a Swedish control survey.
Spectrophotometry at Mölndal showed a distinct bilirubin peak (absorbance, $451 \mathrm{~nm}, 0.043 \mathrm{AU}$; Figure 2B). Of 15 responding laboratories, eight reported that the result indicated SAH if serum bilirubin $<20 \mu \mathrm{mol} / \mathrm{L}$, five reported that the result indicated SAH, 1 reported that the result excluded SAH, and one responded that results without accompanying blood samples were not interpreted. In sum, for a sample with a significant spectrophotometric oxyhaemoglobin peak $8 \mathrm{~h}$ after headache onset, $\mathrm{SAH}$ was reported as the only interpretation by three laboratories and unlikely by three laboratories. A more careful interpretation, that SAH could not be excluded but that a traumatic tap must also be considered, was only reported by 11 of 17 laboratories. The reports were more consistent when the sample was dominated by a significant bilirubin peak, where only 1 of 15 laboratories answered that the results excluded SAH.

\section{Germany, Austria, Switzerland, the Netherlands, and USA}

In the German-speaking countries (Germany, Austria, and Switzerland), the use of absorbance spectrophotometry for SAH diagnostics is still uncommon. Instead, CSF ferritin measurement is the method of choice at most centers because it is easily available on the routine laboratory instruments that already measures CSF total protein, glucose, and lactate. Current guidelines by the German Society of CSF Diagnostics and Clinical Neurochemistry (DGLN) suggest visual inspection including the "threetube method", cell differentiation, and measurement of ferritin when looking for an SAH [52], but measuring the absorbance with a spectrophotometer was recently named as alternative method [45].

In the Netherlands, national guidelines for CSF analyses following SAH are still awaited at time of writing. The Erasmus Medical Center has adopted the UK NEQAS guidelines using Chalmers calculation. A computer-based iterative method to interpret spectrophotometric results (described below) have been developed by Dutch researchers [16]. A recent review from a leading national center on SAH recommended visual analysis [53], which resulted in an educative scientific exchange [54-57].

In the USA, LP is acknowledged for SAH examination (visual inspection and cell counts) after a negative CT scan, but spectrophotometry is still uncommon [58]. There is not a formal quality control program in use for CSF bilirubin testing because it is not a procedure practiced widely in the country. 


\section{Confounding factors}

All CSF analyses described above are subject to confounding factors related to the LP, sample handling, or interpretation.

\section{Timing of the LP}

The timing of the LP in relation to symptom onset has a pivotal impact on all results (Figure 3). CSF production and turnover replaces the entire CSF volume four times each day [59], making it a dynamic compartment. Erythrocytes may be found in the lumbar region approximately $2 \mathrm{~h}$ after an intracranial bleeding [38]. Oxyhaemoglobin also starts to appear around this time point, whereas bilirubin first shows $12 \mathrm{~h}$ after the bleeding [50, 60]. For the detection of oxyhaemoglobin, it is recommended that sampling is done no sooner than $6 \mathrm{~h}$ after symptom onset, when a large enough CSF volume has passed from intracranial areas to the lumbar sack, making the lumbar sample representative. Because the oxyhaemoglobin peak diminishes over time, an isolated oxyhaemoglobin peak without any signs of bilirubin in a sample taken more than $12 \mathrm{~h}$ after symptom onset suggests contamination by a traumatic tap.

\section{Sample transport and storage}

The transport of the sample to the laboratory should be done swiftly, and not in a pneumatic tube, to avoid in vitro haemolysis [61] because this may lead to false-positive results for oxyhaemoglobin and impair the ability to detect bilirubin. At the laboratory, the sample must be centrifuged as soon as possible if a spectrophotometry analysis should be done. If the red cell count is the method in use, centrifugation should not be done. UK guidelines [50] and others [62] suggest centrifugation within $1 \mathrm{~h}$ after

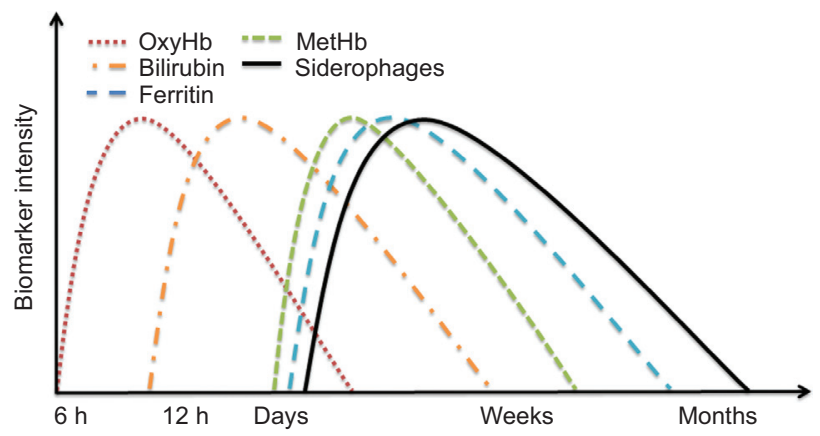

Figure 3 Schematic time-dependent dynamics of CSF biomarkers for SAH. sampling, whereas Swedish laboratories are generally recommended to centrifuge within $30 \mathrm{~min}$. These cutoffs are arbitrary, and in our clinical experience, haemolysis may start even within 30 min in some samples, whereas it may take several hours in other samples. The supernatant should be stored at $4^{\circ} \mathrm{C}$ in the dark until analysis to avoid ultraviolet light-dependent degradation of bilirubin, producing false-negative results [63].

\section{False-positive bilirubin}

Bilirubin may be false positive due to hyperbilirubinemia (a serum sample should therefore be taken together with CSF to allow adjustment for this), significant bloodbrain barrier damage, malignancies, or treatment with rifampicin [28, 64].

\section{Use the least bloody sample}

Although the "three-tube method" is susceptible to false results, serial tubes may be useful because an initial bloody sample portion may be discarded immediately and the least bloodstained sample used for analysis (at the laboratory, red blood cell counts may be done on partial samples from all available tubes, and the sample with the lowest count used for spectrophotometry). A clearing between the first tube and the last tube cannot exclude SAH. In a study by Heasley et al. [65], clearing, defined as a $25 \%$ reduction of RBC between the first tube and the fourth tube, was present even in cases with ruptured aneurysms.

\section{Interpreting the spectrophotometry}

Spectrophotometry is generally appreciated as the gold standard method for CSF analyses of SAH, but there is no definite consensus on curve interpretation, neither in terms of the roles of oxyhaemoglobin vs. bilirubin nor on definitions of pathological cutoffs. One study [17] found low specificity and positive predictive value of spectrophotometry, recommending CT angiography as an alternative. However, such studies have typically not carefully adjusted for the presence of a traumatic tap masking the presence of bilirubin [66]. Gunawardena et al. suggest that patients with oxyhaemoglobin alone and a high suspicion of SAH may benefit from angiography, although the risk for aneurysm is low [67]. A result positive for bilirubin, with or without oxyhaemoglobin, means a high risk for a bleeding aneurysm and should lead to angiography if the 
CSF bilirubin cannot be explained otherwise. According to the UK guidelines, elevated oxyhaemoglobin and bilirubin is reported as consistent with SAH [50], irrespective of CSF protein levels [68]. A problem is that a traumatic tap may lead to increased oxyhaemoglobin and together with either elevated CSF proteins or hyperbilirubinemia may lead to a spectroscopy result that is reported as "consistent with SAH" $[69,70]$. If bilirubin is elevated without a visible oxyhaemoglobin peak and CSF protein levels are raised, meningitis or other diagnoses are more likely [68]. It is therefore important that the clinicians carefully consider the clinical history, serum bilirubin concentrations, CSF protein levels as well as the number of LP attempts.

Visual interpretation of the curve is prone to interobserver variability. Chalmers and Kiley [71] have described a standardized method to calculate NBA using a baseline drawn as tangent to the curve between 360 and $530 \mathrm{~nm}$ and measuring the distances from the baseline to the curve at 415 and $440 \mathrm{~nm}$ to compensate for the oxyhaemoglobin load. The method was later modified by Chalmers [51], using a measurement at $476 \mathrm{~nm}$ only, where the influence from oxyhaemoglobin is so small that the bilirubin measurement does not need compensation. Viljoen et al. [72] have investigated the imprecision when calculating the NBA according to Chalmers' modification. When measuring the within-operator variability, they found a variation of $17 \%$ at the medical decision level suggested by the UK guidelines. To minimize the imprecision, they suggested the use of a software-assisted measurement. Recently, Collet et al. [73] proposed such a method, a webbased software to interpret CSF pigments, that is based on the NEQAS guidelines [73]. For clinicians and laboratory personnel, it is important that they are aware of the variability in the NBA calculation, especially when calculated on a single NBA cutpoint, as recommended in the UK guidelines [72, 74]. An iterative (step-by-step) method, where a computer program calculates the contributions from methaemoglobin, oxyhaemoglobin, and bilirubin from absorbance at 405, 414, and $455 \mathrm{~nm}$, respectively, may be used [16], but it has lower diagnostic accuracy than a derivative method, using a standard of serial dilutions of CSF with known bilirubin concentrations [75], compared with angiography as gold standard.

\section{Towards an optimal CSF biomarker for SAH?}

There is a consensus that spectrophotometry has the highest diagnostic performance of CSF methods for SAH analyses, but visual evaluation of CSF samples is still widely in clinical use in some countries. The major disadvantages of spectrophotometry are equipment cost, the need for trained staff to interpret results, and the lack of a global consensus on the optimal usage of the method. In the absence of compelling study data defining its cost-benefit, the costs involved in purchase of the spectrophotometer system, in the maintenance of the equipment, and the skill level of the individuals who would perform the testing are hard to justify in some countries, including the USA. Bilirubin has a strong positive predictive value for $\mathrm{SAH}$, but relying solely on bilirubin, ignoring oxyhaemoglobin as an indicator of bleeding, requires an approximately 12 - $\mathrm{h}$ wait between symptom debut and CSF tapping, which may be a logistic problem with prolonged stay for the patient at the emergency ward, and in some cases, a medical risk because a delayed investigation increases the risk for an early rebleeding [76, 77]. CSF ferritin is a promising method but carries a risk for false-negative results early after a bleeding. Finally, in rare cases with negative CT scans and CSF examinations, transcranial Doppler sonography and CT/MRI angiography have been reported to be useful [78].

\section{Conclusions}

Visual inspection of CSF for xanthochromia is still frequently used for CSF-based diagnosis of SAH, but it is advised against because spectrophotometric methods have superior diagnostic accuracy. Positive findings of CSF bilirubin in the early stages or of CSF ferritin in the later stages are strong indicators of an intracranial bleeding. A positive finding of CSF oxyhaemoglobin may indicate an intracranial bleeding or a traumatic tap. Rigorous control of confounding factors may reduce the risk for false results.

Studies that compare different methods have not been done, and therefore, it is hard to evaluate what method is preferable. Many factors (confounding factors as well as costs, knowledge of the staff, etc.) have to be accounted for in such a comparison. Today, deciding what method to use in a certain laboratory seems to be dependent on local knowledge, opinions, and thinking. In sum, more of a proven experience rather than evidence decides how the CSF sample will be handled, but guidelines built on research are being used more and more. However, the overall variability described in this review calls for the establishment of a global proficiency testing program for CSF-based SAH diagnostics. 


\section{Conflict of interest statement}

Author's conflict of interest disclosure: The author stated that there are no conflicts of interest regarding the publication of this article.

Research funding: None declared.

\section{References}

1. Feigin VL, Lawes CM, Bennett DA, Anderson CS. Stroke epidemiology: a review of population-based studies of incidence, prevalence, and case-fatality in the late 20th century. Lancet Neurol 2003;2:43-53.

2. Hop JW, Rinkel GJ, Algra A, van Gijn J. Case-fatality rates and functional outcome after subarachnoid hemorrhage: a systematic review. Stroke 1997; $28: 660-4$.

3. Johnston SC, Selvin S, Gress DR. The burden, trends, and demographics of mortality from subarachnoid hemorrhage. Neurology 1998;50:1413-8.

4. Edlow JA, Caplan LR. Avoiding pitfalls in the diagnosis of. subarachnoid hemorrhage. N Engl J Med 2000;342:29-36.

5. de Falco FA. Sentinel headache. Neurol Sci 2004;25:S215-7.

6. Polmear A. Sentinel headaches in aneurysmal subarachnoid. haemorrhage: what is the true incidence? A systematic review. Cephalalgia 2003;23:935-41.

7. Jakobsson KE, Saveland H, Hillman J, Edner G, Zygmunt S, Brandt $\mathrm{L}$, et al. Warning leak and management outcome in aneurysmal subarachnoid hemorrhage. J Neurosurg 1996;85:995-9.

8. Gambhir S, O'Grady G, Koelmeyer T. Clinical lessons and risk factors from 403 fatal cases of subarachnoid haemorrhage. J Clin Neurosci 2009;16:921-4.

9. Mayer PL, Awad IA, Todor R, Harbaugh K, Varnavas G, Lansen TA, et al. Misdiagnosis of symptomatic cerebral aneurysm. Prevalence and correlation with outcome at four institutions. Stroke 1996;27:1558-63.

10. van der Wee N, Rinkel GJ, Hasan D, van Gijn J. Detection of subarachnoid haemorrhage on early CT: is lumbar puncture still needed after a negative scan? J Neurol Neurosurg Psychiatry 1995;58:357-9.

11. Perry JJ, Stiell IG, Sivilotti ML, Bullard MJ, Emond M, Symington C, et al. Sensitivity of computed tomography performed within six hours of onset of headache for diagnosis of subarachnoid haemorrhage: prospective cohort study. BMJ 2011;343:d4277.

12. Morgenstern LB, Luna-Gonzales H, Huber JC Jr, Wong SS, Uthman MO, Gurian JH, et al. Worst headache and subarachnoid hemorrhage: prospective, modern computed tomography and spinal fluid analysis. Ann Emerg Med 1998;32:297-304.

13. van Gijn J, van Dongen KJ. The time course of aneurysmal haemorrhage on computed tomograms. Neuroradiology 1982;23:153-6.

14. Sidman R, Spitalnic S, Demelis M, Durfey N, Jay G. Xanthrochromia? By what method? A comparison of visual and spectrophotometric xanthrochromia. Ann Emerg Med 2005; 46:51-5.

15. Arora S, Swadron SP, Dissanayake V. Evaluating the sensitivity of visual xanthochromia in patients with subarachnoid. hemorrhage. J Emerg Med 2010;39:13-6.
Employment or leadership: None declared. Honorarium: None declared.

Received November 17, 2012; accepted March 12, 2013; previously published online May 9, 2013

16. Duiser HJ, Roelandse FW, Lentjes EG, van Loon J, Souverijn JH, Sturk A. Iterative model for the calculation of oxyhemoglobin, methemoglobin, and bilirubin in absorbance spectra of cerebrospinal fluid. Clin Chem 2001;47:338-41.

17. Wood MJ, Dimeski G, Nowitzke AM. CSF spectrophotometry in the diagnosis and exclusion of spontaneous subarachnoid haemorrhage. J Clin Neurosci 2005;12:142-6.

18. Wick M, Pfister HW. Ferritin and iron metabolism in cerebrospinal fluid (CSF) subarachnoid hemorrhage (SAH). IFCC - WorldLab ‘99, Firenze, Italy, 1999:77.

19. Veuger AJ, Kortbeek LH, Booij AC. Siderophages in differentiation of blood in cerebrospinal fluid. Clin Neurol Neurosurg 1977;80:46-56.

20. Edlow JA, Wyer PC. Evidence-based emergency medicine/clinical question. How good is a negative cranial computed tomographic scan result in excluding subarachnoid hemorrhage? Ann Emerg Med 2000;36:507-16.

21. Mitchell P, Wilkinson ID, Hoggard N, Paley MN, Jellinek DA, Powell T, et al. Detection of subarachnoid haemorrhage with magnetic resonance imaging. J Neurol Neurosurg Psychiatry 2001;70:205-11.

22. Unruptured intracranial aneurysms - risk of rupture and risks of surgical intervention. International Study of Unruptured Intracranial Aneurysms Investigators. N Engl J Med 1998;339:1725-33.

23. Edlow JA, Bruner KS, Horowitz GL. Xanthochromia. Arch Pathol Lab Med 2002;126:413-5.

24. Petzold A, Keir G, Sharpe TL. Why human color vision cannot reliably detect cerebrospinal fluid xanthochromia. Stroke 2005;36:1295-7.

25. Shah KH, Edlow JA. Distinguishing traumatic lumbar puncture from. true subarachnoid hemorrhage. J Emerg Med 2002;23:67-74.

26. Marton KI, Gean AD. The spinal tap: a new look at an old test. Ann Intern Med 1986;104:840-8.

27. Eskey CJ, Ogilvy CS. Fluoroscopy-guided lumbar puncture: decreased frequency of traumatic tap and implications for the assessment of CT-negative acute subarachnoid hemorrhage. AJNR Am J Neuroradiol 2001;22:571-6.

28. Petzold A, Sharpe LT, Keir G. Spectrophotometry for cerebrospinal fluid pigment analysis. Neurocrit Care 2006;4:153-62.

29. Shah KH, Richard KM, Nicholas S, Edlow JA. Incidence of traumatic lumbar puncture. Acad Emerg Med 2003;10:151-4.

30. Petzold A, Keir G, Sharpe LT. Spectrophotometry for xanthochromia. N Engl J Med 2004;351:1695-6.

31. Linn FH, Voorbij HA, Rinkel GJ, Algra A, van Gijn J. Visual inspection. versus spectrophotometry in detecting bilirubin in cerebrospinal. fluid. J Neurol Neurosurg Psychiatry 2005;76:1452-4.

32. Vermeulen M. Subarachnoid haemorrhage: diagnosis and treatment. J Neurol 1996;243:496-501. 
33. Buruma OJ, Janson HL, Den Bergh FA, Bots GT. Blood-stained cerebrospinal fluid: traumatic puncture or haemorrhage? J Neurol Neurosurg Psychiatry 1981;44:144-7.

34. Cruickshank AM. ACP Best Practice No 166: CSF spectrophotometry in the diagnosis of subarachnoid haemorrhage. J Clin Pathol 2001;54:827-30.

35. Chao CY, Florkowski CM, Fink JN, Southby SJ, George PM. Prospective validation of cerebrospinal fluid bilirubin in suspected. subarachnoid haemorrhage. Ann Clin Biochem 2007;44:140-4.

36. Ungerer JP, Southby SJ, Florkowski CM, George PM. Automated. measurement of cerebrospinal fluid bilirubin in suspected. subarachnoid hemorrhage. Clin Chem 2004;50:1854-6.

37. Beetham R. CSF bilirubin-spectrophotometry or direct. measurement? Ann Clin Biochem 2007;44:99-100.

38. Barrows LJ, Hunter FT, Banker BQ. The nature and clinical significance of pigments in the cerebrospinal fluid. Brain 1955;78:59-80.

39. Wahlgren NG, Lindquist C. Haem derivatives in the cerebrospinal fluid after intracranial haemorrhage. Eur Neurol 1987;26:216-21.

40. Trbojevic-Cepe M, Vogrinc Z, Brinar V. Diagnostic significance of methemoglobin determination in colorless cerebrospinal fluid. Clin Chem 1992;38:1404-8.

41. Watson ID, Beetham R, Fahie-Wilson MN, Holbrook IB, O'Connell DM. What is the role of cerebrospinal fluid ferritin in the diagnosis of subarachnoid haemorrhage in computed tomography-negative patients? Ann Clin Biochem 2008;45:189-92.

42. Petzold A, Worthington V, Pritchard C, Appleby I, Kitchen N, Smith $M$. The longitudinal profile of bilirubin and ferritin in. the cerebrospinal fluid following a subarachnoid hemorrhage: diagnostic implications. Neurocrit Care 2009;11:398-402.

43. Petzold A, Worthington V, Appleby I, Kerr ME, Kitchen N, Smith M. Cerebrospinal fluid ferritin level, a sensitive diagnostic test in late-presenting subarachnoid hemorrhage. J Stroke Cerebrovasc Dis 2011;20:489-93.

44. Keir G, Tasdemir N, Thompson EJ. Cerebrospinal fluid ferritin in brain necrosis: evidence for local synthesis. Clin Chim Acta 1993;216:153-66.

45. Tumani H, Petzold A, Wick M, Kuhn HJ, Uhr M, Otto M, et al. [Cerebrospinal fluid-based diagnostics of CT-negative subarachnoid haemorrhage]. Nervenarzt 2010;81:973-9.

46. Marshall RA, Hejamanowski C. Urine test strips to exclude cerebral spinal fluid blood. West J Emerg Med 2011;12:63-6.

47. Page KB, Howell SJ, Smith CM, Dabbs DJ, Malia RG, Porter NR, et al. Bilirubin, ferritin, D-dimers and erythrophages in the cerebrospinal fluid of patients with suspected subarachnoid haemorrhage but negative computed tomography scans. J Clin Pathol 1994;47:986-9.

48. Lang DT, Berberian LB, Lee S, Ault M. Rapid differentiation of subarachnoid hemorrhage from traumatic lumbar puncture using the D-dimer assay. Am J Clin Pathol 1990;93:403-5.

49. UK National External Quality Assessment Scheme for Immunochemistry Working Group. National guidelines for analysis of cerebrospinal fluid for bilirubin in suspected subarachnoid haemorrhage. Ann Clin Biochem 2003;40:481-8.

50. Cruickshank A, Auld P, Beetham R, Burrows G, Egner W, Holbrook I, et al. Revised national guidelines for analysis of cerebrospinal fluid for bilirubin in suspected subarachnoid haemorrhage. Ann Clin Biochem 2008;45:238-44.

51. Chalmers AH. Cerebrospinal fluid xanthochromia testing simplified. Clin Chem 2001;47:147-8.
52. Petereit HF, Sindern E, Wick M. Leitlinien und Methodenkatalog der Deutschen Gesellschaft für Liquordiagnostik und Klinische Neurochemie. Heidelberg: Springer, 2007.

53. van Gijn J, Kerr RS, Rinkel GJ. Subarachnoid haemorrhage. Lancet 2007;369:306-18.

54. Batra YK, Rajeev S. Subarachnoid haemorrhage. Lancet 2007;369:903.

55. Kreitschmann-Andermahr I, Schneider HJ. Subarachnoid haemorrhage. Lancet 2007;369:903-4.

56. Jolobe OM. Subarachnoid haemorrhage. Lancet 2007;369:904.

57. Holbrook I, Beetham R, Cruickshank A, Keir G, Watson I. Subarachnoid haemorrhage. Lancet 2007;369:904.

58. Edlow JA, Panagos PD, Godwin SA, Thomas TL, Decker WW. American College of Emergency P. Clinical policy: critical issues in the evaluation and management of adult patients presenting to the emergency department with acute headache. Ann Emerg Med 2008;52:407-36.

59. Johanson CE, Duncan JA 3rd, Klinge PM, Brinker T, Stopa EG, Silverberg GD. Multiplicity of cerebrospinal fluid functions: new challenges in health and disease. Cerebrospinal Fluid Res 2008;5:10.

60. Morgan CJ, Pyne-Geithman GJ, Jauch EC, Shukla R, Wagner $\mathrm{KR}$, Clark JF, et al. Bilirubin as a cerebrospinal fluid marker of sentinel subarachnoid hemorrhage: a preliminary report in pigs. J Neurosurg 2004;101:1026-9.

61. Wenham PR, Hanson T, Ashby JP. Interference in spectropho: tometric analysis of cerebrospinal fluid by haemolysis induced. by transport through a pneumatic tube system. Ann Clin Biochem 2001;38:371-5.

62. Graves P, Sidman R. Xanthochromia is not pathognomonic for subarachnoid hemorrhage. Acad Emerg Med 2004;11:131-5.

63. Foroughi M, Parikh D, Wassell J, Hatfield R. Influence of light and time on bilirubin degradation in CSF spectrophotometry for subarachnoid haemorrhage. Br J Neurosurg 2010;24:401-4.

64. Lo BM, Quinn SM. Gross xanthochromia on lumbar puncture may not represent an acute subarachnoid hemorrhage. Am J Emerg Med 2009;27:621-3.

65. Heasley DC, Mohamed MA, Yousem DM. Clearing of red blood cells in lumbar puncture does not rule out ruptured aneurysm in patients with suspected subarachnoid hemorrhage but negative head CT findings. AJNR Am J Neuroradiol 2005;26:820-4.

66. Watson ID, Beetham R, Keir G, Cruickshank AM, Holbrook IB, Fahie-Wilson MN, et al. Cerebrospinal fluid spectrophotometry of bilirubin, not the Xanthochromic Index, for the detection of CT-negative sub-arachnoid haemorrhage. J Clin Neurosci 2007;14:608-9.

67. Gunawardena H, Beetham R, Scolding N, Lhatoo SD. Is cerebrospinal fluid spectrophotometry useful in CT scan-negative suspected subarachnoid haemorrage? Eur Neurol 2004;52:226-9.

68. Cruickshank AM, Proteins UNSAGfEQAoC, Biochemistry. Revision of national guidelines for cerebrospinal fluid analysis in suspected subarachnoid haemorrhage. Ann Clin Biochem 2008;45:236-7.

69. Sulaiman RA, Gama R. Pitfalls in cerebrospinal fluid spectroscopy results for the diagnosis of subarachnoid haemorrhage. Br J Neurosurg 2010;24:726.

70. Griffiths MJ, Ford C, Gama R. Revised national guidelines for the analysis of cerebrospinal fluid for bilirubin in suspected 
subarachnoid haemorrhage: interpret with caution. J Clin Pathol 2009;62:1052.

71. Chalmers AH, Kiley M. Detection of xanthochromia in cerebrospinal fluid. Clin Chem 1998;44:1740-2.

72. Viljoen A, Walker SW, Walker KS, Twomey PJ. Imprecision of cerebrospinal fluid net bilirubin absorbance. Clin Chem 2004;50:1266-8.

73. Collet N, Garcelon N, Robbe V, Lucas-Clerc C, Cuggia M, Bendavid C. Bioinformatic software for cerebrospinal fluid spectrophotometry in suspected subarachnoid haemorrhage. Ann Clin Biochem 2012;49:177-83.

74. Viljoen A, Walker KS, Ho C, Twomey PJ. Analysis of cerebrospinal fluid for suspected subarachnoid haemorrhage is improved by built-in spectrophotometer software. J Clin Pathol 2006;59:667.

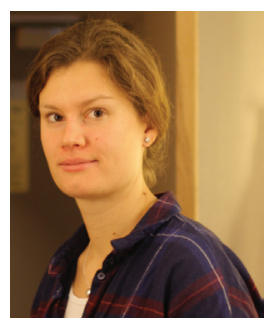

Karin Nagy has just received her Medical Degree from the University of Gothenburg and is currently on her internship to become a licensed physician. The review "Cerebrospinal fluid analyses for the diagnosis of subarachnoid haemorrhage and experience from a Swedish study" is a part of the research she did for her medical thesis.

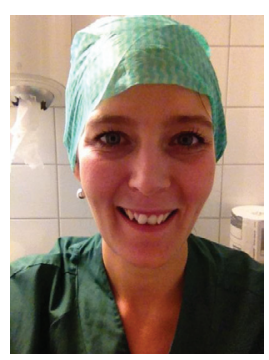

Ina Skagervik is a Neurosurgical Resident since 2009 at Sahlgrenska University Hospital, Gothenburg, Sweden. She received her Medical Degree in 2006. She is clinically active in various neurosurgical fields in order to complete her specialist competence in neurosurgery in 2014. She recently started research in different methods of diagnosing subarachnoid haemorrhage in cerebrospinal fluid.
75. O'Connell DM, Watson ID. Definitive angiographic detection of subarachnoid haemorrhage compared with laboratory assessment of intracranial bleed in CT-negative patients. Ann Clin Biochem 2003;40:269-73.

76. Fujii Y, Takeuchi S, Sasaki O, Minakawa T, Koike T, Tanaka R. Ultra-early rebleeding in spontaneous subarachnoid hemorrhage. J Neurosurg 1996;84:35-42.

77. Mark DG, Pines JM. The detection of nontraumatic subarachnoid hemorrhage: still a diagnostic challenge. Am J Emerg Med 2006;24:859-63.

78. Eggers C, Liu W, Brinker G, Fink GR, Burghaus L. Do negative CCT and CSF findings exclude a subarachnoid haemorrhage? A retrospective analysis of 220 patients with subarachnoid haemorrhage. Eur J Neurol 2011;18:300-5.

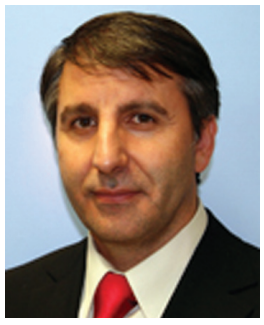

Hayrettin Tumani received his Medical Degree from the University of Göttingen in 1991. He received board certification in Neurology in 1999 and completed his habilitation thesis, "Brain-specific proteins in CSF: physiology and clinical relevance”, in 2001. Dr. Tumani is a Professor of Neurology and the Director of the Multiple Sclerosis Center at the Department of Neurology, University School of Medicine, Ulm, Germany. He is also the Director of the CSF Laboratory, and past president of the German Society of CSF Analysis and Clinical Neurochemistry (DGLN). His scientific areas of interest include diagnostic and prognostic biomarkers in multiple sclerosis as well as in neurodegenerative diseases such as amyotrophic lateral sclerosis.

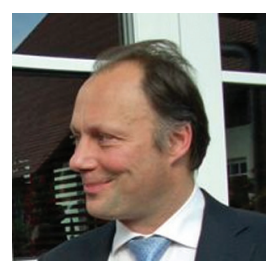

Axel Petzold graduated from the Medical University of Freiburg (Germany). He received his MD (summa cum laude) in Experimental Ophthalmology with Ted Sharpe (University of Freiburg) and his PhD in Biochemistry with Ed Thompson (University College London). He trained as a neurologist in France (Lyon), Germany (Munich), and the UK (London). He now works as Consultant Neurologist at the VU Medical Center Amsterdam and as an Honorary Senior Lecturer at the UCL Institute of Neurology, Queen Square. His research interest is on axonal degeneration mainly in multiple sclerosis and optic nerve disease and also other diseases and models he can learn from. 


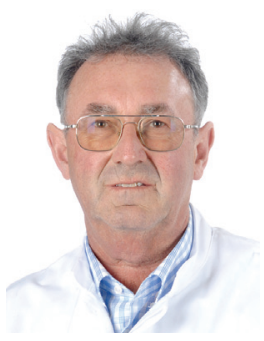

Manfred Wick graduated from the Medical School of Ludwig Maximilian University of Munich. He is now a consultant laboratory physician for the Department of Neurology, Klinikum Großhadern, University of Munich. Since 1994, he is a senior physician and assistant laboratory director with special interest in immunology, CSF analysis, and iron metabolism. He is a member of the Board of the German Society for CSF Analysis and Clinical Neurochemistry, and since 2002, he is a member of the Educational Commission.

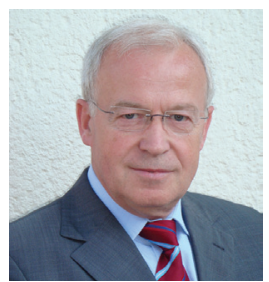

Hans-Jürgen Kühn was born in 1944 in Sommerfeld (now called Lubsko). Since 1945, he has been is living in Leipzig. After school, he studied chemistry at the University of Leipzig and finished it 1969, receiving a "Diplomchemiker" degree. In 1970, he started his career in the field of clinical chemistry. From 1975 to 2009, he was the head of the CSF Laboratory at the Medical Department of the University of Leipzig. In 1982, he defended a dissertation about copper metabolism in Wilson disease. He is a member of the German Society of Clinical Chemistry (DGKL) and foundation and honor member of the German Society of CSF Diagnosis and Clinical Neurochemistry (DGLN).

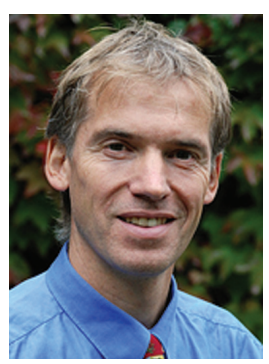

Manfred Uhr is the assistant medical director and head of the Neurological Outpatient Clinic, head of the Department of Clinical Neurophysiology (EEG, evoked potentials, EMG, sonography), and head of the Neurochemical Laboratory comprising the conduction of CSF analyses, therapeutic drug monitoring, hormone detection, and further laboratory parameters required for the diagnosis of neurological and psychiatric diseases at the Max Planck Institute of Psychiatry. One of the aims of the research group is to investigate the influence of the blood-brain barrier on the etiology and therapy of neurological and psychiatric diseases. The group's interest has been focused on transport proteins carrying their substrates against a concentration gradient from the brain back into the blood, thus influencing the endocrinological regulatory circuit and the pharmacokinetics of drugs acting on the CNS.

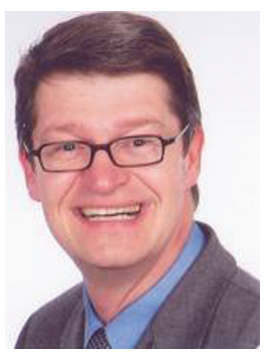

Axel Regeniter, MD, PhD, is the deputy director of clinical chemistry at Basel's University Hospital. His area of expertise is protein biochemistry, where analysis results are usually complex, interrelated, and difficult to interpret. He developed a knowledge-based system that combines text output with visually oriented report forms to transfer the information from the laboratory to the clinical ward most effectively. The original system for the differentiation of proteinuria is available in five languages and in routine use in over 100 laboratories. Ongoing development led to the creation of "Visual MDI Lablink", which applies this to many different areas of laboratory medicine, for instance, cerebrospinal fluid analysis, protein electrophoresis and immunofixation, neurological auto antibodies, chimerism, or the assessment of nutritional status. In his spare time, he loves to participate in outdoor activities like golf and skiing.

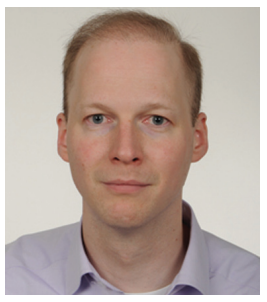

Johannes Brettschneider received his medical training at the University of Tuebingen, Germany. He is a neurologist with special interests in electromyography, palliative care, and geriatrics. In 2011, he was a visiting associate professor at the University of Pennsylvania Center of Neurodegenerative Disease Research (CNDR).

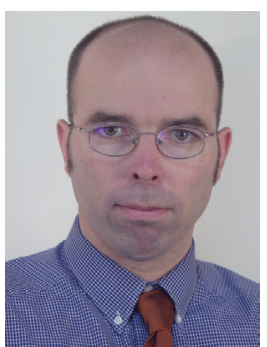

Markus Otto is a professor of neurology at the University of Ulm, the elected president of German Society of CSF Diagnostics and Neurochemistry (www.dgln.de), and speaker of the German Consortium for Frontal Lobar Degenerations (www.ftld.de), which is supported 
by the German Ministry of Science and Development. His focus lies on the development and evaluation of methods and markers for the early and differential diagnosis of neurodegenerative diseases and in the investigation of the roles of these proteins in models of these diseases.

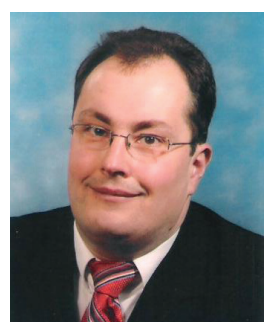

Jörg Kraus, MD, completed his primary and secondary education in Weiden, Germany, and studied medicine at the University of Regensburg (November 1990 to September 1992) and the JustusLiebig University of Giessen (October 1992 to November 1997). He completed his MD thesis in 2000. The title of his thesis was "ICAM 1, ICAM 3, and CD45RA in cerebrospinal fluid and blood as activity parameters in multiple sclerosis". He subsequently received his "Venia Legendi" at the Paracelsus Private Medical University of Salzburg, having completed his habilitation thesis "The impact of interferon-treatment on the blood-brain barrier”. Since 1995, Dr. Kraus has been working at the MS outpatient departments of three different university hospitals.

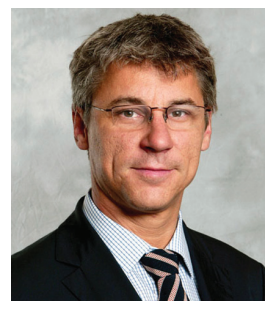

Florian Deisenhammer is presently a professor of neurology at the University of Innsbruck in Austria. After graduating in 1989 from the University of Vienna Medical School, he finished his training in clinical neurology at Innsbruck Medical University in 1996 and became a senior staff member since then. He is the director of the Neuroimmunological Laboratory, and his clinical work focuses on stroke and neuroimmunology, particularly multiple sclerosis. His main scientific interest is MS therapy monitoring, specifically antibodies against interferons and other biomarkers in CSF and blood. He is a member of various international and national societies including the Austrian Medical Society, the International Society of Neuroimmunology, the EFNS Task Force on Beta Interferon Neutralizing Antibodies, board member of the German Society of Neurochemistry and CSF Diagnostics, the Task Force on CSF, and member of the editorial board of the Journal Multiple Sclerosis and Related Disorders (MSARD).

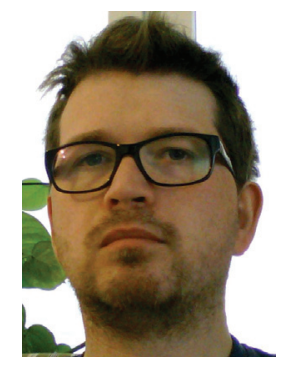

Ronald Lautner is a medical doctor and researcher. He received his medical education at Innsbruck Medical University, Austria. He is now a physician in specialist training (clinical chemistry) at Sahlgrenska University Hospital, and a PhD student at the research team on "Neurochemical Pathogenesis and Diagnostics" at the Department of Neuroscience and Physiology, University of Gothenburg, Sweden. His research is focused on cerebrospinal fluid biomarkers in the clinical diagnosis of Alzheimer disease and other brain disorders, including neuroinflammation and brain injury.

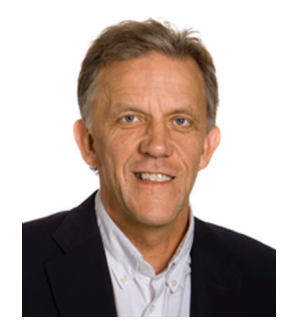

Kaj Blennow took his medical degree (MD) in 1984 and holds a specialist competence in both general psychiatry and in clinical chemistry. He is the head of the Clinical Neurochemistry Lab at Sahlgrenska University Hospital, Gothenburg, Sweden, and a professor of clinical neurochemistry at the Sahlgrenska Academy, Mölndal campus at the University of Gothenburg, Sweden. His major research areas are cerebrospinal fluid biochemical markers, clinical proteomics, and the neurochemical pathogenesis of Alzheimer disease and other brain disorders. Blennow has published more than 550 original research papers and 80 review articles in peerreviewed journals and has an ISI Web of Knowledge $\mathrm{H}$-index of 71 (March 2013). He has received a number of scientific awards, such as the CINP Award (1992), the IPA Research Award (1993), the Alois Alzheimer Research Award (2001), the ECNP Clinical Research Award (2010), and the Henry Wisniewski Lifetime Achievement Award in Alzheimer's Disease Research (2011).

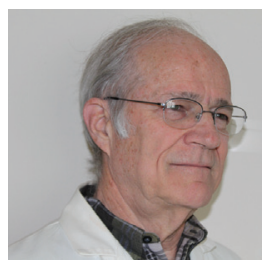

Leslie Shaw is a professor at the Department of Pathology and Lab Medicine at Hospital of the University of Pennsylvania. In 1968, he received his $\mathrm{PhD}$ in biochemistry. He has received several awards, honors, and membership in honorary societies since then. Leslie Shaw has published more than 200 original research papers and review articles in peer-reviewed journals. His main scientific 
interests are biomarkers in Alzheimer disease. He is also a member of the NCCLS Area Committee on Toxicology and Drug Monitoring (1989 to present).

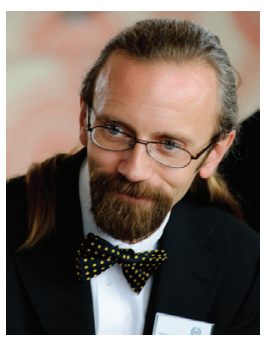

Henrik Zetterberg has a background in molecular biology and clinical chemistry and has spent the last 10 years focusing on the development of biomarkers for Alzheimer disease and other brain disorders. His research is translational and the biomarkers are evaluated in cell and animal models, as well as in longitudinal studies of patients and healthy individuals. He has developed new diagnostic tests for Alzheimer disease, as well as new preclinical models, and has shown that myeloid pathology precedes $\tau$ pathology around 5 years during the Alzheimer disease process in humans, that altered amyloid homeostasis in the brain is evident already in presymptomatic stages of the disease, and that the diagnostic usefulness of AD biomarkers decreases with age due to increased prevalence of preclinical Alzheimer neuropathology.

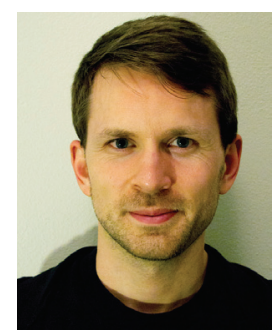

Niklas Mattsson is a medical doctor and researcher. He is a certified specialist in clinical chemistry and is currently doing postdoctoral research at the University of California San Francisco in the group of Michael W. Weiner. His research is focused on biomarkers for neurological diseases, especially cerebrospinal fluid and imaging markers for neurodegenerative diseases. He received his medical education in Lund and Gothenburg (Sweden) and did his doctorate studies at the University of Gothenburg, studying cerebrospinal fluid biomarkers for axonal degeneration and amyloid metabolism in Alzheimer disease, neuroinflammation, and inborn errors of metabolism. 